\title{
The Artin-Lang property for normal real analytic surfaces
}

\author{
By Carlos Andradas, Antonio Díaz-Cano and Jesús M. Ruiz at Madrid
}

\begin{abstract}
We solve the 17th Hilbert Problem and prove the Artin-Lang property for normal real analytic surfaces. Then we deduce that the absolute (resp. relative) holomorphy ring of such a surface consists of all bounded (resp. locally bounded) meromorphic functions.
\end{abstract}

\section{Introduction and main results}

Let $X$ be an (affine) real analytic surface, that is, a real analytic set of dimension 2 defined in some open set $\Omega \subset \mathbb{R}^{n}$; this $n$ we call the embedding dimension of $X$. The sheaf of germs of analytic functions on $\Omega$ is denoted by $\mathcal{O}_{\Omega}$, and its global sections are the global analytic functions on $\Omega$, which form the ring $\mathcal{O}(\Omega)$. Contrarily to the complex case, the sheaf $\mathscr{J}_{X}$ of germs of analytic functions vanishing on $X$ need not be coherent.

However, it is so if the surface is normal, which we assume henceforth. The surface $X$ is equiped with the (restriction of the) coherent sheaf $\mathcal{O}_{X}=\mathcal{O}_{\Omega} / \mathscr{J}_{X}$. The global sections of the sheaf $\mathcal{O}_{X}$ are the global analytic functions on $X$, and form the ring $\mathcal{O}(X)=\mathcal{O}(\Omega) / \mathscr{J}(X)$. In case $X$ is irreducible $\mathscr{J}(X)$ is a prime ideal, and $\mathcal{O}(X)$ is a domain. In general, $X$ decomposes into a locally finite collection of irreducible components $X_{i}$. Since a normal surface is locally irreducible, its irreducible components are its connected components, and consequently $\mathcal{O}(X)$ is the product of all $\mathcal{O}\left(X_{i}\right)$ 's.

The sheaf $\mathscr{M}_{X}$ of meromorphic functions of $X$ is defined by its stalks: $\mathscr{M}_{X, x}$ is the total ring of fractions of $\mathcal{O}_{X, x}$. Since $X$ is coherent, the ring of global sections of $\mathscr{M}_{X}$ is the total ring of fractions $\mathscr{M}(X)$ of $\mathcal{O}(X)$; that is the ring of meromorphic functions on $X$. If $X$ is irreducible, $\mathscr{M}(X)$ is the quotient field of the domain $\mathcal{O}(X)$. In the general case $\mathscr{M}(X)$ is the product of the fields of meromorphic functions $\mathscr{M}\left(X_{i}\right)$ of the irreducible components $X_{i}$ of $X$.

The goal of this paper is to solve several problems concerning meromorphic functions on the normal surface $X$. Namely, we will first prove:

Authors partially supported by DGICYT, PB98-0756-C02-01, second author also by the Universidad de Castilla-La Mancha. 
Theorem 1 (17th Hilbert Problem). Every non-negative meromorphic function on $X$ is a sum of $p$ squares of meromorphic functions, where $p$ is a positive integer that depends solely on the embedding dimension of $X$.

The second half of this statement is quantitative in nature, and amounts to say that the Pythagoras number of $\mathscr{M}(X)$ is finite, bounded by a function of the embedding dimension. The first half is qualitative, and through Artin-Schreier theory means that a meromorphic function on $X$ is positive somewhere if and only if it is positive in every ordering of $\mathscr{M}(X)$. Actually, we will get more:

Theorem 2 (Artin-Lang Property). The condition for finitely many meromorphic functions on $X$ to be positive at some point of $X$ is that they are positive in some ordering of $\mathscr{M}(X)$.

Secondly, we will consider two different types of meromorphic functions, in connection with the so-called holomorphy rings of $X$. Let us suppose $X$ is irreducible, so that $\mathscr{M}(X)$ is a field. Then, the absolute holomorphy ring $\mathscr{H}(X)$ is the intersection of all real valuation rings of $\mathscr{M}(X)$, and the relative holomorphy ring $\mathscr{H}^{\prime}(X)$ is the intersection of those that contain $\mathcal{O}(X)$. Clearly $\mathscr{H}(X) \subset \mathscr{H}^{\prime}(X)$, and in some way the two rings are extremal. Both rings are Prüfer rings and are related to the study of sum of squares and other important questions ([3]). We will prove the following:

Theorem 3. (a) The absolute holomorphy ring $\mathscr{H}(X) \subset \mathscr{M}(X)$ is the ring of bounded meromorphic functions of $\mathscr{M}(X)$.

(b) The relative holomorphy ring $\mathscr{H}^{\prime}(X) \subset \mathscr{M}(X)$ is the ring of locally bounded meromorphic functions of $\mathscr{M}(X)$.

Of course both holomorphy rings coincide if $X$ is compact. In this case the result was already known, and the Krull dimension of the holomorphy ring is the dimension of $X$ (see [1]). Here we will see that if $X$ is not compact both holomorphy rings have infinite Krull dimension, by exhibiting infinite rank real valuations.

All these questions have an old history: the local case (germs) was settled back in 1974 by Risler, and the global case for compact $X$ by Ruiz and Jaworski independently in 1985; also in the compact case, holomorphy rings were described in 1999 by AbánadesJoglar-Ruiz. Some refinements under milder compactness assumptions are due to Castilla and Jaworski in 1991. Without compactness, the problems are solved for non-singular curves and non singular surfaces in several papers by Jaworski, Andradas-Becker and Castilla from 1982 till 2000. The reader can find more details of this progress in [2], Notes to Ch. VIII.

We stress that here we deal with a normal surface $X$, possibly singular, possibly non compact. The restriction to the normal case is usually solved by normalization. However this has some difficulties in the real analytic case. Of course, one has to deal with coherent surfaces to get a birational surjective normalization, but the crucial difficulty here refers to embeddings: the normalization of an affine surface may not be affine again. Thus, our results in this paper extend readily via normalization to every coherent real analytic surface $X$ whose normalization $X^{\prime}$ is affine, which happens when the local embedding dimension of 
$X^{\prime}$ is bounded. This holds true if: (i) the singularities of $X$ have bounded multiplicities, (ii) $X^{\prime}$ has finitely many singularities, or (iii) $X$ is algebraic.

We further remark that the union of all dimension 1 components of $X$ is a non singular curve, on which every non negative analytic function is a sum of two squares ([8]). This means that we can forget this one-dimensional part and suppose the normal surface has dimension 2 everywhere, that is, it is a pure surface. However, we do not assume our surface is irreducible. In fact, the case when $X$ has infinitely many components will be important to bound the Pythagoras number.

Part of this paper was written during a stay of the second author at the Institute de Recherche Mathematique de Rennes, a research place all of us have had the occasion to enjoy. We thank Prof. M. Coste and the Institute for a warm welcoming and a fruitful atmosphere.

\section{Multiplicities along an irreducible curve}

Let $X \subset \Omega \subset \mathbb{R}^{n}$ be a normal pure surface. Let $Y \subset X$ be an irreducible curve.

For every function $f \in \mathcal{O}(X)$ we define its multiplicity $m_{Y}(f)$ along $Y$ as follows. Fix a point $x \in Y \backslash \operatorname{Sing}(X)$ (always possible as $\operatorname{Sing}(X)$ is discrete). Since $\mathcal{O}_{X, x}$ is a unique factorization domain, the ideal $\mathscr{J}_{Y, x}$ is principal, say $\mathscr{J}_{Y, x}=h_{x} \mathcal{O}_{X, x}$, and we have $f_{x}=v_{x} h_{x}^{\alpha_{x}}$ for some $v_{x} \in \mathcal{O}_{X, x} \backslash \mathscr{J}_{Y, x}$ and some integer $\alpha_{x} \geqq 0$. This integer $\alpha_{x}$ is to be the multiplicity $m_{Y}(f)$. Let us show that this definition is consistent.

Firstly, $\alpha_{x}$ does not depend on the generator: assume $f_{x}=w_{x} g_{x}^{\beta_{x}}$ for another generator $g_{x}$ and $w_{x} \in \mathcal{O}_{X, x} \backslash \mathscr{J}_{Y, x}$. If, say, $\alpha_{x} \leqq \beta_{x}$, we get $h_{x}=u_{x} g_{x}$ for a unit $u_{x}$ of $\mathcal{O}_{X, x}$, and $w_{x} g_{x}^{\beta_{x}}=v_{x}\left(u_{x} g_{x}\right)^{\alpha_{x}}$, so that

$$
w_{x} g_{x}^{\beta_{x}-\alpha_{x}}=v_{x} u_{x}^{\alpha_{x}} \notin \mathscr{J}_{Y, x},
$$

which means $\beta_{x}-\alpha_{x}=0$.

Secondly, $\alpha$ is constant on some (local) branch of $Y_{x}$. Indeed, for $y$ close to $x$ we have $f_{y}=v_{y} h_{y}^{\alpha_{x}}$ and $h_{y}$ is a generator of $\mathscr{J}_{Y, x}$ (curves are coherent). Furthermore, since $v_{x} \notin \mathscr{J}_{Y, x}$, $v$ cannot vanish on all branches of $Y_{x}$, so that $v(y) \neq 0$ for $y$ close to $x$ in that branch. We conclude $\alpha_{x}=\alpha_{y}$.

Next, we claim:

There exists a global analytic function $g \in \mathcal{O}(X)$ which vanishes on $Y$ and whose germ at $x$ generates $\mathscr{J}_{Y, x}$ for all $x \in Y$ off a discrete set $D \subset Y$.

Indeed, choose any regular point $a$ of $Y$ off $\operatorname{Sing}(X)$. By Cartan's Theorem A, the ideal $\mathscr{J}_{Y, a}$ is generated by a finite number of global functions $f_{1}, \ldots, f_{r} \in \mathcal{O}(X)$ which vanish on $Y$, and at least one of the germs $f_{i, a}$ does not belong to $\mathscr{J}_{Y, a}^{2}$, say this is true for $i=1$. Then we put $g=f_{1}$, so that $g_{a}=v_{a} h_{a}$ with $v_{a} \notin \mathscr{J}_{Y, a}$. As above, we see that $a$ is isolated in $Y \cap\{v=0\}$, hence $g_{x}$ generates $\mathscr{J}_{Y, x}$ for all $x \in Y \backslash\{a\}$ close enough to $a$. Now, consider the coherent sheaf of ideals

$$
\begin{array}{r}
\mathscr{I}_{x}=\left(g_{x} \mathcal{O}_{X, x}: \mathscr{J}_{Y, x}\right), \quad x \in X, \\
\text { Brought to you by | Swets (Swets) } \\
\text { Authenticated | 172.16.1.226 } \\
\text { Download Date | 3/9/12 10:56 AM }
\end{array}
$$


and notice that $\mathscr{I}_{x}=\mathcal{O}_{X, x}$ if and only if $g_{x}$ generates $\mathscr{J}_{Y, x}$. In this situation, the support

$$
\operatorname{supp}\left(\mathcal{O}_{X} / \mathscr{I}\right)=\left\{x \in X: g_{x} \text { does not generate } \mathscr{J}_{Y, x}\right\}
$$

is a closed analytic set $Y^{\prime}$ and does not contain $Y$. As $Y$ is irreducible, $D=Y \cap Y^{\prime}$ is a discrete set. So $g_{x}$ generates $\mathscr{J}_{Y, x}$ for all $x \in Y \backslash D$, and the claim is proved.

After this, we come back to the definition of $m_{Y}(f)$. We fix a regular point $a$ of $Y$ off $\operatorname{Sing}(X) \cup D$. We have $f_{a}=v_{a} g_{a}^{\alpha_{a}}$ with $v_{a} \notin \mathscr{J}_{Y, a}$. Again, $v_{x}$ is a unit for $a \neq x \in Y \backslash D$ close to $a$ and consequently

$$
\alpha_{x}=\alpha_{a}, \quad g_{x}^{\alpha_{a}}\left|f_{x}, \quad f_{x}\right| g_{x}^{\alpha_{a}}
$$

Now, we consider the coherent sheaf of ideals $\mathscr{I}$ of $\mathcal{O}_{X}$ given by

$$
\mathscr{I}_{x}=\left(g_{x}^{\alpha_{a}}: f_{x}\right) \cap\left(f_{x}: g_{x}^{\alpha_{a}}\right), \quad x \in X
$$

The support

$$
\operatorname{supp}\left(\mathcal{O}_{X} / \mathscr{I}\right)=\left\{x \in X: g_{x}^{\alpha_{a}} \nmid f_{x} \text { or } f_{x} \nmid g_{x}^{\alpha_{a}}\right\}
$$

is an analytic set $Y^{\prime \prime}$ that does not contain $Y$, hence $D^{\prime}=Y^{\prime \prime} \cap Y$ is discrete, and we deduce that $\alpha_{x}=\alpha_{a}$ for every $x \in Y$ off $\operatorname{Sing}(X) \cup D \cup D^{\prime}$. The conclusion follows from this and the fact that for every $x, \alpha$ is constant on some branch of $Y_{x}$.

Remarks 1.1. (1) The preceding arguments show that: If $m_{Y}(f)=\alpha$, there is a discrete set $E$ such that $f_{x} / g_{x}^{\alpha}$ is a unit for $x \in Y \backslash E$.

(2) From the preceding remark, we see that $m_{Y}(f)$ is odd if and only if $f$ changes sign at every point of $Y$. In that case, we will simply say that $f$ changes sign at $Y$.

(3) The function $g$ we have constructed vanishes on $Y$, but can, and sometimes must, have additional zeros. The usual example is a nontrivial circle $Y$ in a torus $X$ : since $Y$ does not disconnect $X$, no function with zero set $Y$ can change sign. For a less immediate example, consider the cone $X \subset \mathbb{R}^{3}: x^{2}+y^{2}=z^{2}$ and the line $Y \subset X: z=x, y=0$, which disconnects $X$. We have for instance $g=y$, but this $g$ vanishes also on the line $z=-x, y=0$. Even we can find another function not vanishing on this second line, as $g^{\prime}=y+(z-x)^{2}$, but then some other additional zeros appear.

However, we can always modify $g$ to avoid any prescribed curve $Y^{\prime}$ which meets $Y$ at isolated points. Indeed, if $h \in \mathcal{O}(X)$ and $f \in \mathcal{O}(X)$ have zero sets $Y$ and $Y^{\prime}$ respectively, then the function $\tilde{g}=f g+h^{2}$ has the same properties as $g$ and does not vanish on $Y^{\prime} \backslash Y$.

(4) Put in algebraic form, we have proved that the localization $\mathcal{O}(X)_{\mathfrak{p}}$ at the prime ideal $\mathfrak{p}=\mathscr{J}(Y)$ is a discrete valuation ring, with uniformizer $g$, and $m_{Y}$ is exactly the valuation associated to it. Note that this is a real valuation, since its residue field is the quotient field of $\mathcal{O}(X) / \mathfrak{p}$, which is the field of meromorphic functions of $Y$. 
(5) Of course, we can define the multiplicity of a meromorphic function $h=f / g$ by $m_{Y}(h)=m_{Y}(f)-m_{Y}(g)$.

(6) Fixed any integer $\alpha \geqq 0$ there is a global analytic function $f$ with multiplicity $\alpha$ along $Y$. With the notations above, take $f=g^{\alpha}$.

\section{Functions with prescribed multiplicities}

Let us fix the normal pure surface $X$ as before, and consider an analytic curve $Y \subset X$ without isolated points, whose irreducible components are denoted $Y_{i}(i \in I)$. Fix a sequence of integers $m_{i} \geqq 0(i \in I)$. We discuss here the problem of finding an analytic function $f \in \mathcal{O}(X)$ with multiplicity $m_{i}$ along $Y_{i}(i \in I)$.

Proposition 2.1. Suppose that all $m_{i}$ 's are even. Then there is $h \in \mathcal{O}(X)$ which is a sum of squares such that $m_{Y_{i}}(h)=m_{i}$ and $\mathscr{Z}(h)=\bigcup_{m_{i}>0} Y_{i}$.

Proof. Choose for each $i \in I$ a uniformizer $g_{i}$ of the valuation $m_{Y_{i}}$ which does not vanish on any $Y_{j}, j \neq i$ (Remarks $\left.1.1(3)\right)$, and a sum of squares $h_{i}$ which is an equation for $Y_{i}$. Set $m_{i}=2 n_{i}$ and consider the sheaf of ideals given by

$$
\mathscr{I}_{x}=\left(\prod_{i \mid x \in Y_{i}} g_{i, x}^{n_{i}}, \prod_{i \mid x \in Y_{i}} h_{i, x}^{n_{i}}\right),
$$

for $x \in X$. To see this sheaf $\mathscr{I}$ is well defined and coherent, one takes a neighborhood $U$ of $a \in X$ such that all $Y_{i}$ 's that meet $U$ pass through $a$, and checks that $\mathscr{I}$ is generated in $U$ by the two functions $\prod_{a \in Y_{i}} g_{i}^{n_{i}}, \prod_{a \in Y_{i}} h_{i}^{n_{i}}$. This also implies that the sheaf is globally generated by finitely many global sections $f_{1}, \ldots, f_{r}$ (see [6]). Now, let $h=f_{1}^{2}+\cdots+f_{r}^{2}$, so that $\mathscr{Z}(h)=\bigcup_{n_{i}>0} Y_{i}$. We also know that for any $x \in Y_{i} \backslash \bigcup_{j \neq i} Y_{j}$ off a suitable discrete set, the germ $g_{i, x}$ generates $\mathscr{J}_{Y_{i}, x}$ and consequently

$$
\left(g_{i, x}^{n_{i}}\right)=\mathscr{I}_{x}=\left(f_{1, x}, \ldots, f_{r, x}\right) .
$$

Thus, by the properties of valuations,

$$
m_{Y_{i}}\left(f_{\ell}\right) \geqq n_{i} \geqq \min _{k} m_{Y_{i}}\left(f_{k}\right),
$$

and so $n_{i}=\min _{k} m_{Y_{i}}\left(f_{k}\right)$. Since $m_{Y_{i}}$ is a real valuation, we conclude

$$
m_{Y_{i}}(h)=2 \min _{k} m_{Y_{i}}\left(f_{k}\right)=2 n_{i}
$$

Next, we consider the case where all multiplicities are 1:

Lemma 2.2. There is a function $g \in \mathcal{O}(X)$ such that $m_{Y_{i}}(g)=1$ for all $i \in I$. In particular, $g$ changes sign at all $Y_{i}$ 's. 
Proof. We define the coherent sheaf of ideals $\mathscr{I}$ as in the proof of Proposition 2.1, here with all exponents $n_{i}=1$, and find global sections $f_{1}, \ldots, f_{r}$ that generate $\mathscr{I}$. In particular, all the $f_{k}$ 's vanish on $Y$. For each $k=1, \ldots, r$, we consider the set $I_{k}$ of the indices $i \in I$ such that $f_{k}$ is the first function with multiplicity 1 along $Y_{i}$ :

$$
m_{Y_{i}}\left(f_{1}\right)>1, \ldots, m_{Y_{i}}\left(f_{k-1}\right)>1, \quad m_{Y_{i}}\left(f_{k}\right)=1 .
$$

We notice that the $I_{k}$ 's are a partition of $I$. Now, set $Z_{k}=\bigcup_{i \in I_{k}} Y_{i}$, choose an equation $h_{k}$ of $Z_{k}$ which is a sum of squares, and define $g_{k}=f_{k}+h_{k}$. Since $m_{Y_{i}}$ is a valuation, we have

$$
m_{Y_{i}}\left(g_{k}\right) \geqq \min \left\{m_{Y_{i}}\left(f_{k}\right), m_{Y_{i}}\left(h_{k}\right)\right\}
$$

with equality if the multiplicities of $f_{k}$ and $h_{k}$ are distinct. Since the valuation is real, $m_{Y_{i}}\left(h_{k}\right)$ is an even number, and we see that $m_{Y_{i}}\left(g_{k}\right)=1$ if $Y_{i} \subset Z_{k}$, and $m_{Y_{i}}\left(g_{k}\right)=0$ otherwise. But $Y_{i}$ is contained in a unique $Z_{k}$, and we conclude that the product $g_{1} \cdots g_{r}$ has multiplicity 1 along $Y_{i}$. Thus $g=g_{1} \cdots g_{r}$ is the function we were looking for.

Finally, we turn to the general result:

Proposition 2.3. There is $f \in \mathcal{O}(X)$ such that $m_{Y_{i}}(f)=m_{i}$ for all $i \in I$.

Proof. We write $m_{i}=2 n_{i}$ or $m_{i}=2 n_{i}+1$ according to the parity. By 2.1 , there is a sum of squares $h \in \mathcal{O}(X)$ such that $m_{Y_{i}}(h)=2 n_{i}$ for all $i \in I$, and $\mathscr{Z}(h)=\bigcup_{n_{i}>0} Y_{i}$. By 2.2, there is $g \in \mathcal{O}(X)$ with $m_{Y_{i}}(g)=1$ when $m_{i}$ is odd. Also, we take a sum of squares $e$ which is an equation of the analytic curve $Y^{\prime}$ consisting of the $Y_{i}$ 's with odd $m_{i}$ and the $Y_{i}$ 's on which $g$ does not vanish. With this choice, $f=h(g+e)$ is the function we sought. Indeed,

$$
m_{Y_{i}}(f)=m_{Y_{i}}(h)+m_{Y_{i}}(g+e)
$$

and an easy discussion of cases shows that $m_{Y_{i}}(g+e)=0$ or 1 , according to the parity of $m_{i}$.

\section{The 17th Hilbert problem}

In this section we solve the 17 th Hilbert problem in a technical form that will be essential to deduce later the Artin-Lang property.

Let $X \subset \Omega \subset \mathbb{R}^{n}$ be a normal pure surface. Let $f \in \mathcal{O}(X)$ be an analytic function and $Z$ its zero set. For each irreducible component $Y$ of dimension 1 of $Z$ the valuation $m_{Y}$ gives multiplicities along $Y$. In particular, $f$ changes sign along $Y$ if and only if $m_{Y}(f)$ is odd, and the union of the $Y^{\prime}$ 's for which this is the case is denoted $Z^{0}$. In addition, $f$ may change sign at some of its isolated zeros, and these are collected in a discrete set denoted by $D^{0}$.

The important fact is the following: 
Proposition 3.1. There is an analytic function $\tilde{f} \in \mathcal{O}(X)$ such that:

(a) The zero set of $\tilde{f}$ is $Z^{0} \cup D^{0}$ and $\tilde{f}$ generates the sheaf of ideals $\mathscr{J}_{Z^{0}}$ off a discrete set.

(b) $h_{0}^{2} f=\left(h_{1}^{2}+\cdots+h_{r}^{2}\right) \tilde{f}$, with $h_{0}, \ldots, h_{r} \in \mathcal{O}(X)$; moreover, the zero set of $h_{0}$ is discrete.

In particular, $\tilde{f}$ and $f$ change sign at the same points and $\tilde{f}$ changes sign at every zero.

Proof. We can suppose that $X$ is closed in $\mathbb{R}^{n}$, after embedding $\Omega$ as a closed submanifold of a bigger affine space (Grauert's theorem); this guarantees that discrete sets in $X$ are also discrete in $\mathbb{R}^{n}$, a detail that will be crucial later. Now, by 2.1 , with a suitable choice of even multiplicities along the irreducible components of $Z$, we can find a sum of squares of analytic functions, $h$, such that off a discrete set $\left\{x_{i}: i \in I\right\}$ the meromorphic function $f / h$ is analytic, generates $\mathscr{J}_{Z^{0}}$ and its zero set is $Z^{0}$. This is close to the $\tilde{f}$ we seek, but not it yet and some modifications must be made.

To start with, consider the coherent sheaf $(h: f)$. This sheaf is generated in a neighborhood of each $x_{i}$ by finitely many sections $\delta_{1}, \ldots, \delta_{r}$. By the standard sum of squares trick, $f_{x_{i}} / h_{x_{i}}=g_{x_{i}} / \delta_{x_{i}}$ for $\delta=\sum_{k} \delta_{k}^{2}$ and some $g_{x_{i}}$. Furthermore, $x_{i}$ is an isolated zero of $\delta$. For, suppose there is $x \neq x_{i}$ arbitrarily close to $x_{i}$ with $\delta(x)=0$. Then, all $\delta_{k}$ 's vanish at $x$, and since the ideal $\left(h_{x}: f_{x}\right)$ is generated by them, it contains no unit. This means that $f_{x} / h_{x}$ is not analytic, a contradiction. Adding the square of an equation of $X_{x_{i}}$, we can extend $\delta_{x_{i}}$ to a sum of squares of analytic germs in $\mathbb{R}_{x_{i}}^{n}$ that vanishes only at $x_{i}$ : call $\tilde{\delta}_{x_{i}}$ that extension. The ideals $\mathscr{I}_{x_{i}}=\left(\tilde{\delta}_{x_{i}}\right)$ glue to define a locally free coherent sheaf of ideals $\mathscr{I}$ of $\mathcal{O}_{\mathbb{R}^{n}}$, whose zero set is $\left\{x_{i}: i \in I\right\}$. Since $H^{1}\left(\mathbb{R}^{n}, \mathbb{Z}_{2}\right)=0$, all locally free sheaves are free, and $\mathscr{I}$ has a global generator $\Delta$; this means that each germ $\Delta_{x_{i}} / \tilde{\delta}_{x_{i}}$ is a unit. This $\Delta$ is a non-negative analytic function on $\mathbb{R}^{n}$ with zeros the $x_{i}$ 's, and $f^{\prime}=\Delta f / h$ is analytic. By [4], Th. 1 and its proof, $\Delta$ is a sum of squares

$$
\Delta=\left(g_{1} / g_{0}\right)^{2}+\cdots+\left(g_{s} / g_{0}\right)^{2}
$$

where each $g_{i}$ is an analytic function and the zeros of $g_{0}$ are again the $x_{i}$ 's. Since $\Delta$ has discrete zero set, $f^{\prime}$ and $f / h$ have the same properties off a discrete set. Furthermore, we have

$$
g^{2} f=g_{0}^{2} g h f^{\prime}, \quad \text { where } g=g_{1}^{2}+\cdots+g_{s}^{2} \text { has discrete zero set. }
$$

Thus, we are left with the condition that the zero set of $f^{\prime}$ be $Z^{0} \cup D^{0}$. In fact, since $X$ is pure dimensional, the above formula shows that $f^{\prime}$ changes sign at a point $x$ if and only if so does $f$, hence the zero set of $f^{\prime}$ contains $Z^{0} \cup D^{0}$, and we have to discard a discrete set of points $\left\{y_{j}: j \in J\right\}$ at which $f^{\prime}$ vanishes but does not change sign. This requires some additional manipulations.

First, by the 17th Hilbert problem for germs ([2], VIII.5.8), we find a sum of squares $\lambda_{y_{j}} \in \mathcal{O}_{X_{y_{j}}}$ which vanishes only at $y_{j}$ such that $\varepsilon_{j} \lambda_{y_{j}} f_{y_{j}}^{\prime}$ is a sum of squares (with $\varepsilon_{j}= \pm 1$ ). Let $\bar{\lambda}_{y_{j}}$ be a sum of squares in $\mathbb{R}_{y_{j}}^{n}$ which vanishes only at $y_{j}$ and extends $\lambda_{y_{j}}$. As we did 
before for $\Delta$, now we find a non-negative analytic function $\Lambda$ on $\mathbb{R}^{n}$ with discrete zero set $\left\{y_{j}: j \in J\right\}$, hence a sum of squares of meromorphic function germs whose denominators do not vanish off $\left\{y_{j}: j \in J\right\}$, and such that each quotient $\Lambda_{y_{j}} / \lambda_{y_{j}}$ is a unit. By these conditions, we can replace $f^{\prime}$ by $f^{*}=\Lambda f^{\prime}$ and have $f_{y_{j}}^{*}=\Lambda_{y_{j}} f_{y_{j}}^{\prime}=u_{y_{j}} \lambda_{y_{j}} f_{y_{j}}^{\prime}$, where $u_{y_{j}}$ is a unit. Hence $f_{y_{j}}^{*}$ is a sum of squares of analytic germs, and as we found $\Delta$ and $\Lambda$ above, we now find a non-negative analytic function $\eta$ of $\mathbb{R}^{n}$ which does not vanish off $\left\{y_{j}: j \in J\right\}$ and such that each quotient $\eta_{y_{j}} / f_{y_{j}}^{*}$ is a unit; furthermore, $\eta$ is a sum of squares of meromorphic functions with denominators vanishing only at the $y_{i}$ 's. Finally, we can replace $f^{*}$ by $\tilde{f}=f^{*} / \eta$, and this $\tilde{f}$ is analytic and the $y_{j}$ 's have been discarded from its zero set. The proof is thus complete.

To justify our former assertion that the preceding result is a solution to the 17th Hilbert problem, we deduce the qualitative part of the 17th Hilbert problem:

Corollary 3.2. Let $X$ be a normal surface. Then, every non-negative analytic function $f$ on $X$ is a sum squares of meromorphic functions.

Proof. As was explained in the introduction, we can assume $X$ is pure. Then, we apply 3.1 to $f$, and notice that $\tilde{f}$ does not vanish, because $f$ does not change sign anywhere. It follows $\tilde{f}$ is $>0$ and a square. We are done.

For the quantitative part we have:

Corollary 3.3. Let $X$ be a normal surface. Then, the Pythagoras number of $\mathscr{M}(X)$ has an upper bound $p(n)$ that depends solely on the embedding dimension $n$ of $X$.

Proof. Suppose that there is a sequence of normal surfaces $X_{k} \subset \Omega_{k} \subset \mathbb{R}^{n}$ whose rings of meromorphic functions have Pythagoras numbers $p_{k} \rightarrow \infty$. Pick any family of open balls $D_{k}$ whose closures do not meet; since $\mathbb{R}^{n}$ is analytically diffeomorphic to the open ball of radius 1, we may assume that $\Omega_{k} \subset D_{k}$. By hypothesis, in every $\Omega_{k}$ there is an analytic function $F_{k}$ whose restriction $f_{k}$ to $X_{k}$ is non-negative and cannot be represented as a sum of less than $p_{k}$ squares of meromorphic functions on $X_{k}$. Now, $X=\bigcup_{k} X_{k}$ is a normal surface in $\Omega=\bigcup_{k} \Omega_{k} \subset \mathbb{R}^{n}$, and the $F_{k}$ 's define an analytic function $F$ in $\Omega$ whose restriction to $X$ is non-negative. By the qualitative result 3.2, $f$ is a sum of squares of meromorphic functions, say of $p$ squares, which by restriction to every $X_{k}$ give a representation of $f_{k}$ as a sum of $p$ squares of meromorphic functions. But then $p \geqq p_{k}$ for all $k$, which is a contradiction.

\section{The Artin-Lang property}

Clearly, it is enough to prove Theorem 2 of the introduction for an irreducible normal surface $X$.

Proof. Suppose that $f_{1}, \ldots, f_{r} \in \mathcal{O}(X)$ are positive at some point $x \in X$. Then, the $f_{i}$ 's are positive in any ordering centered at $x$. This is the easy implication. For the converse, let us suppose that the set $f_{1}>0, \ldots, f_{r}>0$ is empty. We claim: 
The closed semianalytic set $T$ defined by $\widetilde{f_{1}} \geqq 0, \ldots, \widetilde{f_{r}} \geqq 0, \widetilde{f_{1} f_{2}} \geqq 0, \ldots, \widetilde{f_{r-1} f_{r}} \geqq 0$ is discrete.

To show this, we first note that by the construction of free square functions, $\widetilde{f_{1}}>0, \ldots, \widetilde{f_{r}}>0$ must be empty as $f_{1}>0, \ldots, f_{r}>0$ is, and consequently, $T$ is contained in the union of the zero sets of the $\widetilde{f_{i}}$, s. Here we notice the reason to consider the $\widetilde{f_{i} f_{j}}$ 's: if both $f_{i}$ and $f_{j}$ change sign along an irreducible curve $Y$, then so do $\widetilde{f}_{i}$ and $\widetilde{f}_{j}$, but not their product, so that $\widetilde{f_{i} f_{j}}$ does not vanish on $Y$. Now suppose $T$ has dimension 1 . Then, moving along a 1-dimensional branch of $T$, we can find an open set $B$ such that:

(i) $B$ is contained in the regular locus of $X$.

(ii) $B \cap T$ is a smooth connected curve, that decomposes $B$ in two connected components $U$ and $V$.

(iii) For each $g=\widetilde{f}_{i}$ or $\widetilde{f_{i}} f_{j}, B \cap\{g=0\}$ is either a smooth curve or empty.

After this preparation, since $\widetilde{f}_{1}>0, \ldots, \widetilde{f}_{r}>0$ is empty, there must be some $\widetilde{f}_{i}$ that vanishes exactly on $B \cap T$, hence changes sign, say it is $>0$ on $U$ and $<0$ on $V$. But then again some other $\widetilde{f}_{j}$ must vanish on $B \cap T$ and be $<0$ on $U,>0$ on $V$. Hence $\widetilde{f_{i}} f_{j}$ does not vanish on $B \cap T$, and is $<0$ on $B$. But $\widetilde{f_{i} f_{j}}$ is $\geqq 0$ on $T$, a contradiction. This completes the proof of the claim.

Now, by Grauert's embedding theorem, we can suppose $X$ embedded in $\mathbb{R}^{n}$ with a global analytic equation $H$, and that $F_{i}, F_{i j}$ are analytic extensions of $\widetilde{f_{i}}, \widetilde{f_{i} f_{j}}$ to $\mathbb{R}^{n}$. Let $T=\left\{a_{k}: k \geqq 1\right\}$ and for each $k$ we pick an open set $U_{k}$ with $U_{k} \cap T=\left\{a_{k}\right\}$. We consider the quadratic polynomial $Q_{k}=\left\|x-a_{k}\right\|^{2}$, so that

$$
U_{k} \cap\left\{F_{i} \geqq 0, F_{i j} \geqq 0, H=0, Q_{k} \neq 0\right\}=\emptyset .
$$

Hence, by the Positivstellensatz for analytic germs ([2], VIII.5.6), after shrinking $U_{k}$ we find analytic functions $A_{k, v}, G_{k}$ on $U_{k}$ such that

$$
-Q_{k}^{2 s_{k}}=\sum_{v} A_{k, v} F_{1}^{v_{1}} \cdots F_{r}^{v_{r}} F_{12}^{v_{12}} \cdots F_{r-1 r}^{v_{r-1 r}}+G_{k} H
$$

where the $A_{k, v}$ 's are sums of squares and the exponents $v_{i}, v_{i j}$ are either 0 or 1 . Next, using Cartan's Theorem B, we find global analytic functions $B_{v}, G$ on $\mathbb{R}^{n}$ such that for every $k$ it holds

$$
B_{v} \equiv A_{k, v}+Q_{k}^{2 s_{k}+1} \quad \bmod \mathrm{m}_{k}^{4 s_{k}+3} \quad \text { and } \quad G \equiv G_{k} \quad \bmod \mathrm{m}_{k}^{4 s_{k}+3}
$$

where $\mathfrak{m}_{k}$ is the maximal ideal of the point $a_{k}$ (in the stalk $\mathcal{O}_{\mathbb{R}^{n}, a_{k}}$ ). We now consider the following analytic function on $\mathbb{R}^{n}$ :

$$
P=\sum_{v} B_{v} F_{1}^{v_{1}} \cdots F_{r}^{v_{r}} F_{12}^{v_{12}} \cdots F_{r-1 r}^{v_{r-1 r}}+G H
$$

We have, maybe after shrinking the $U_{k}$ 's, 
(i) $P \leqq 0$ on $U=\bigcup_{k} U_{k}$, because at each $a_{k}$ the initial form of $P$ is $-Q_{k}^{2 s_{k}}$, and

(ii) each $B_{v}$ is $\geqq 0$ on $U$ and $a_{k}$ is an isolated zero of $B_{v}$. Indeed, near $a_{k}$ we have $B_{v}=A_{k, v}+D_{v}$, with

$$
D_{v} \equiv Q_{k}^{2 s_{k}+1} \bmod \mathrm{m}_{k}^{4 s_{k}+3}
$$

whence the initial form of $D_{v}$ is $Q_{k}^{2 s_{k}+1}$, which implies that $D_{v}$ is non-negative and $a_{k}$ is an isolated zero of $D_{v}$. Since $A_{k, v}$ is a sum of squares the assertion follows.

Finally, we consider the restriction to $X$ :

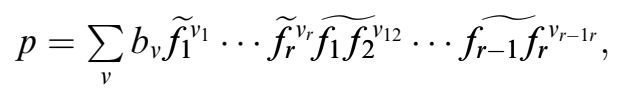

where $p=P \mid X$, and $b_{v}=B_{v} \mid X$ for every $v$.

After this preamble, suppose there is some ordering $\alpha$ of the field $\mathscr{M}(X)$ such that $f_{i}(\alpha)>0$ for all $i$ 's. By construction of the free square functions, we have $\widetilde{f}_{i}(\alpha)>0$, $\widetilde{f_{i} f_{j}}(\alpha)>0$ for all $i, j$ 's. Clearly, we only need to prove

$$
p(\alpha)<0, \quad b_{v}(\alpha)>0,
$$

to get a contradiction. But this comes from the following lemma:

Let $h_{1}, \ldots, h_{s} \in \mathcal{O}(X)$ be positive in an ordering $\alpha$ of $\mathscr{M}(X)$. If $g \in \mathcal{O}(X)$ is $\geqq 0$ on some neighborhood of $\left\{h_{1} \geqq 0, \ldots, h_{s} \geqq 0\right\}$, then $g$ is positive in $\alpha$.

We take a free square function $\tilde{g}$. By the construction of this function, $\tilde{g}$ is $>0$ on $\left\{h_{1} \geqq 0, \ldots, h_{r} \geqq 0\right\}$, and from [2], VIII.4.7-4.8 we see that $\tilde{g}$ is positive in $\alpha$. Hence $g$ is positive too.

\section{Holomorphy rings}

Now that we have solved the 17th Hilbert problem and obtained the Artin-Lang property, we can deduce the direct description of the holomorphy rings in Theorem 3 of the introduction. Again, we only have to prove it for an irreducible normal real analytic surface $X$.

Proposition 5.1. The absolute holomorphy ring $\mathscr{H}(X)$ is the ring of all meromorphic functions bounded on $X$.

Proof. Suppose a meromorphic function $f / g$ is not bounded on $X$ : for every integer $k \geqq 1$ there is a point $x_{k} \in X$ such that $g\left(x_{k}\right) \neq 0$ and $f\left(x_{k}\right)^{2} / g\left(x_{k}\right)^{2}>k$. Since we can center a total ordering of the field $\mathscr{M}(X)$ at every $x_{k}$, the constructible set $T_{k}$ defined in the real spectrum of $\mathscr{M}(X)$ by the condition $f^{2}>\mathrm{kg}^{2}$ is not empty. But that real spectrum is compact, and $T_{k} \supset T_{k+1}$, so that $\bigcap_{k} T_{k} \neq \emptyset$. If $\alpha$ is a total ordering in that intersection, we have $f^{2} / g^{2}>k$ in $\alpha$ for every $k$, and the convex hull of $\mathbb{R}$ in $\mathscr{M}(X)$ with respect to $\alpha$ is 
a real valuation ring that does not contain $f / g$. Conversely, if $f^{2} / g^{2} \leqq k$ on $X$ for some $k \geqq 1$, then $k g^{2}-f^{2} \geqq 0$ on $X$, and by Hilbert's 17 th problem (3.2), $k g^{2}-f^{2} \geqq 0$ is a sum of squares in $\mathscr{M}(X)$. Hence $k g^{2}-f^{2} \geqq 0$ is positive in all orderings of $\mathscr{M}(X)$, that is, $f^{2} / g^{2}$ is bounded by $k$ in all of them. Now if $V$ is a real valuation ring, it is convex with respect to some ordering, and contains $k$, hence contains $f^{2} / g^{2}$. We are done.

Similarly, we have:

Proposition 5.2. The relative holomorphy ring $\mathscr{H}^{\prime}(X)$ is the ring of all meromorphic functions locally bounded on $X$.

Proof. Let $f / g$ be a meromorphic function which is not locally bounded on $X$ : there is a point $x_{0} \in X$ and a sequence $x_{k} \in X$ convergent to $x_{0}$ such that $f\left(x_{k}\right)^{2} / g\left(x_{k}\right)^{2}>k$. Now we work with germs at $x_{0}$. The set germ $\left\{f^{2}-k g^{2} \geqq 0\right\}$ contains the sequence $x_{\ell}$ for $\ell \geqq k$, hence it is not empty. By the properties of semianalytic germs ([2], VIII.2.11) the constructible set $T_{k}$ defined by the condition $f^{2}-k g^{2} \geqq 0$ in the real spectrum of the ring $\mathcal{O}\left(X_{x_{0}}\right)$ contains prime cones $\alpha$ of dimension 2. Again by the compactnes of the real spectrum, we deduce that there is such an $\alpha$ that makes $f^{2}-k g^{2} \geqq 0$ for all $k \geqq 1$, that is $f^{2} / g^{2} \geqq k$ for all $k \geqq 1$. Let $\mathfrak{p} \subset \mathcal{O}_{X, x_{0}}=\mathcal{O}\left(X_{x_{0}}\right)$ be the support of $\alpha$, so that we get an inclusion $\mathcal{O}(X) \subset \mathcal{O}\left(X_{x_{0}}\right) / \mathfrak{p}$, and $\alpha$ induces a total ordering in $\mathscr{M}(X)$. Finally, the convex hull of $\mathbb{R}$ with respect to that total ordering is a real valuation that specializes to $x_{0}$, but does not contain the function $\mathrm{f} / \mathrm{g}$.

Conversely, suppose $f / g$ is locally bounded, and let $V$ be a real valuation that contains $\mathcal{O}(X)$. We pick an ordering $\alpha$ of $\mathscr{M}(X)$ compatible with $V$. Next we can suppose that $X \subset \mathbb{R}^{n}$ and $n$ is the embedding dimension, so that $X$ is not contained in any hyperplane $H \subset \mathbb{R}^{n}$; as $X$ is irreducible, this means that $\operatorname{dim}(H \cap X) \leqq 1$. Next, we consider the following global semianalytic sets:

$$
Z_{\sigma} \subset \mathbb{R}^{n}: h_{\sigma, 1}=\sigma_{1} \sin \left(x_{1}\right)>0, \ldots, h_{\sigma, n}=\sigma_{n} \sin \left(x_{n}\right)>0,
$$

where $\sigma=\left(\sigma_{1}, \ldots, \sigma_{n}\right)$ is any choice of signs \pm 1 . Clearly, each $Z_{\sigma}$ is a discrete union of open polycylinders whose closures are disjoint, and $T=\mathbb{R}^{n} \backslash \bigcup_{\sigma} Z_{\sigma}$ is a discrete union of hyperplanes. By the remark above on the embedding dimension, we find that $X \cap T$ has dimension $\leqq 1$, and no $h_{\sigma, i}$ vanishes on $X$. Thus, for a unique $\sigma$, the functions $h_{\sigma, 1}, \ldots, h_{\sigma, n}$ are positive in $\alpha$. Now, the semianalytic set $S=X \cap Z_{\sigma}$ is a countable union of open sets $S_{k}$ with disjoint compact closures. Then, since $f / g$ is locally bounded, it is bounded on each $S_{k}$, say by $\lambda_{k}$. Next, we produce a continuous function $L$ which is $\equiv 2 \lambda_{k}$ on $S_{k}$, and by approximation, an analytic function $M \in \mathcal{O}(X)$ which is $>\lambda_{k}$ on $S_{k}$. If $M-f^{2} / g^{2}$ is negative in $\alpha$, by the Artin-Lang property the set $S \cap\left\{g^{2} M-f^{2}<0\right\}$ would not be empty, against the construction. If $M=f^{2} / g^{2}$, then $f^{2} / g^{2} \in \mathcal{O}(X) \subset V$. Finally, if $M-f^{2} / g^{2}$ is positive in $\alpha$, since $M \in \mathcal{O}(X) \subset V$ and $V$ is convex with respect to $\alpha, f^{2} / g^{2} \in V$. In any case, since $V$ is integrally closed, $f / g \in V$, and we are done.

The dimension of holomorphy rings. Suppose $X$ is not compact. Let $Y \subset X$ be a real irreducible curve. We choose in $Y$ a discrete infinite sequence of regular points $\left(a_{\ell}\right)$ of $X$, and through each $a_{\ell}$ another irreducible curve $X_{\ell} \subset X$. This can be done to have at each 
$a_{\ell}$ local coordinates $\left(x_{\ell}, y_{\ell}\right)$ of $X$ whose zero set germs $x_{\ell}=0$ and $y_{\ell}=0$ are, respectively, $X_{\ell}$ and $Y$. Now we define a total ordering $\alpha$ using any fixed ultrafilter $\Lambda$ of natural numbers which does not contain finite sets: an analytic function $f$ is positive in $\alpha$ if there is $L \in \Lambda$ such that for every $\ell \in L$ we have an expansion

$$
f\left(x_{\ell}, y_{\ell}\right)=\left(c_{s} y_{\ell}^{s}+\cdots\right) x_{\ell}^{r}+\cdots \in \mathbb{R}\left\{x_{\ell}, y_{\ell}\right\}
$$

with $c_{s}>0$. Notice that the norm $\|x\|^{2}$ is bigger than any real number in this ordering. Once $\alpha$ is defined, consider the convex hulls $W$ of $\mathbb{R}$ in $\mathscr{M}(X)$ and $V$ of $\mathcal{O}(X)$ in $\mathscr{M}(X)$. These are two real valuation rings of $\mathscr{M}(X)$ compatible with $\alpha$ : $W$ is minimal and $V$ is minimal containing $\mathcal{O}(X)$.

(i) Construction of an infinite chain between $W$ and $V$. For every $d \geqq 1$ denote by $\exp _{d}$ the $d$-th iteration of the exponential function, and consider the ring $W_{d}$ consisting of all $h \in V$ such that a bound of the form

$$
|h(x)| \leqq c \exp _{d}\left(\|x\|^{2 N}\right), \quad c>0, N \text { integer } \geqq 1,
$$

holds on some semianalytic set $\left\{f_{1}>0, \ldots, f_{r}>0\right\}$ with the $f_{i}$ 's positive in $\alpha$. Since a bound

$$
\exp _{d+1}\left(\|x\|^{2}\right) \leqq c \exp _{d}\left(\|x\|^{2 N}\right), \quad c>0, N \text { integer } \geqq 1
$$

can hold only if $\|x\|^{2} \leqq \rho$ for some $\rho$, we see that $\exp _{d+1}\left(\|x\|^{2}\right) \in W_{d+1} \backslash W_{d}$, so that the chain is infinite.

(ii) Construction of an infinite chain over $V$. Let $S_{d} \subset V$ consist of all $f \in V$ such that for some constant $c>0$ and some $L \in \Lambda$ we have $m_{X_{\ell}}(f) \leqq c \ell^{d}$ for all $\ell \in L$. This set is multiplicative, and we consider the ring of fractions $V_{d}=V\left[S_{d}^{-1}\right]$. We claim that the inclusion $V_{d} \subset V_{d+1}$ is strict. For, pick an analytic function $f \in \mathcal{O}(X)$ with $m_{X_{\ell}}(f)=\ell^{d+1}$ for every $\ell \geqq 1$ (2.3). Clearly $f \in S_{d+1}$ hence $1 / f \in V_{d+1}$. Suppose now that $1 / f \in V_{d}$, and so there are $g \in V, h \in S_{d}$ with $f(g / h)=1$, that is $f g=h$. One easily checks that, $g$ being bounded by some analytic function, there is $L \in \Lambda$ such that $m_{X_{\ell}}(g) \geqq 0$ for all $\ell \in L$, and by the definition of $S_{d}$ and the choice of $f$ we conclude

$$
\ell^{d+1} \leqq m_{X \ell}(f)+m_{X \ell}(g)=m_{X \ell}(h) \leqq c \ell^{d}
$$

for some $c>0$ and infinitely many $\ell$ 's, which is impossible.

Remark 5.3. The dimension of Prüfer rings like our holomorphy rings, is used to bound the minimal number $\mu$ of generators for finitely generated ideals: in fact $\mu \leqq \operatorname{dim}+1$ ([7]). Thus, if $X$ is compact we get $\mu \leqq 3$, but if $X$ is not compact we cannot deduce any bound. However, by the general properties of holomorphy rings (see [3]), $\mu$ is bounded by the Pythagoras number of $\mathscr{M}(X)$. Since this Pythagoras number is finite (3.3), we conclude that $\mu$ is finite too. For instance ([8] or [4]), if $X$ is a compact non-singular analytic surface, $p=3$, which gives the same bound $\mu \leqq 3$ as above, and if $X$ is a non-compact non-singular analytic surface, $p=2$, and this gives the even better bound $\mu \leqq 2$. 


\section{References}

[1] M. A. Abánades, N. Joglar-Prieto, J. M. Ruiz, Bounded meromorphic functions on compact real analytic sets, J. Pure Appl. Algebra 142 (1999), 1-11.

[2] C. Andradas, L. Bröcker, J. M. Ruiz, Constructible sets in real geometry, Ergeb. Math. 33, Springer-Verlag, 1996.

[3] E. Becker, The real holomorphy ring and sums of $2 n$-th powers, in: Géométrie algébrique réelle et formes quadratiques, Proceedings, Rennes 1981, Springer Lect. Notes Math. 959 (1982), 139-181.

[4] J. Bochnak, W. Kucharz, M. Shiota, On equivalence of ideals of real global analytic functions and the 17 th Hilbert problem, Invent. Math. 63 (1981), 403-421.

[5] A. Castilla, Artin-Lang property for analytic manifolds of dimension two, Math. Z. 217 (1994), 5-14.

[6] S. Coen, Sul rango dei fasci coerenti, Boll. U. Mat. Ital. 22 (1967), 373-383.

[7] R. C. Heitmann, Generating ideals in Prüfer domains, Pacific J. Math. 62 (1976), 117-126.

[8] P. Jaworski, Positive definite analytic functions and vector bundles, Bull. Ac. Polonaise Sc. XXX (1982), 501-506.

[9] P. Jaworski, The 17-th Hilbert problem for noncompact real analytic manifolds, Springer Lect. Notes Math. 1524 (1991), 289-295.

Departamento de Algebra, Facultad de Matematicas, Universidad Complutense, 28040 Madrid, Spain e-mail: carlos_andradas@mat.ucm.es e-mail: antonio_diaz@mat.ucm.es

Eingegangen 12. November 2001, in revidierter Fassung 26. März 2002 